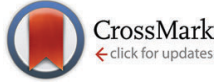

Cite this: J. Mater. Chem. C, 2015 3, 10277

Received 20th May 2015, Accepted 3rd September 2015

DOI: $10.1039 / c 5 t c 01445 j$

www.rsc.org/MaterialsC

\section{Construction of multi-layered white emitting organic nanoparticles by clicking polymers $\dagger$}

\author{
H. Keita, ${ }^{\text {ab }}$ B. Guzelturk, ${ }^{c}$ J. Pennakalathil, ${ }^{a}$ T. Erdem, ${ }^{c}$ H. V. Demir ${ }^{\text {bcd }}$ and \\ D. Tuncel*ab
}

\begin{abstract}
A series of blue, green and red emitting polymers that are appropriately functionalized with alkyne and azide functional groups have been prepared and clicked together to construct bi-layered and tri-layered white emitting core-shell type nanoparticles. Here the use of these organic hetero-nanoparticles as colour converters to realize a white light-emitting diode platform acquiring a colour quality comparable to the existing phosphor-based ones was also demonstrated.
\end{abstract}

\section{Introduction}

White organic light emitting diodes (WOLEDs), either in pixelated arrays or over large areas have attracted great attention from both the scientific community and industry owing to their superior properties potentially for the next-generation of curved-panel displays and surface lighting applications. ${ }^{1-3}$ Due to their low-cost, highefficiency, flexible and eco-friendly qualities, current research efforts have been devoted to the synthesis of novel materials for WOLEDs to reduce global energy consumption. ${ }^{4,5}$ Since Kido et al. reported multilayer WOLEDs, several strategies are now being employed to generate light covering the visible range of the spectrum as much as possible. ${ }^{6-9}$ To obtain emission across the visible spectrum, a set of different fluorophores are utilized, each with distinct emission colours. These typically involve two (blue and green/yellow) or three (blue, green and red) fluorophores. ${ }^{10-13}$

Recent decades have witnessed the introduction of a number of electro and photoactive conjugated polymers, such as polythiophene (PT), poly ( $p$-phenylenevinylene) (PPV), and polyfluorene (PF). Polyfluorene and its derivatives stand out as suitable materials for polymer light emitting diodes (PLEDs) due to their high quantum efficiencies, superior thermal stabilities and the availability of

\footnotetext{
${ }^{a}$ Department of Chemistry, Bilkent University, 06800 Ankara, Turkey

${ }^{b}$ UNAM-National Nanotechnology Research Center, and Institute of Materials

Science and Nanotechnology, Bilkent University, Ankara 06800, Turkey. E-mail: dtuncel@fen.bilkent.edu.tr

${ }^{c}$ Departments of Electrical and Electronics Engineering and Physics, Bilkent University, 06800 Ankara, Turkey

${ }^{d}$ School of Electrical and Electronic Engineering, School of Physical and Mathematical Sciences, Nanyang Technological University, Singapore

$\dagger$ Electronic supplementary information (ESI) available: All experimental details, SEM and TEM images of nanoparticles, and DLS histograms. See DOI: 10.1039/ c5tc01445j
}

the hydrogen on the 9th position of fluorene for functionalization. ${ }^{10-17}$ As a blue emitter with high quantum efficiency, polyfluorene serves as a donor to other lower energy acceptor molecules mainly through non-radiative energy transfer. ${ }^{15-17}$ Non-radiative energy transfer or Förster resonance energy transfer (FRET) is an efficient and rapid process which is aided by the coupled dipole-dipole interaction between donor and acceptor molecules. ${ }^{18}$ Numerous studies also investigated energy transfer in hybrid systems involving donor polymer molecules and acceptor dye molecules for various applications. ${ }^{19,20}$ Since synthetic procedures such as copolymerization can have an undesirable effect on the emission characteristics of the original conjugated polymer, a novel approach to harness and tune the distinct emission of different fluorophores for OLEDs has long being sought. ${ }^{17,21-23}$ Foulger et al. demonstrated emission colour tuning in hybrid bi-polymer nanoparticles for optoelectronic and imaging applications. ${ }^{24}$

Taking advantage of the efficient energy transfer and emission colour tuning in bi-polymer nanostructures, we present tunable white-emitting conjugated polymer nanoparticles (CPNs). ${ }^{25-28}$ The emission properties can be controlled both by varying the acceptor concentration and nanostructure design. Recent studies in our group showed an efficient energy transfer in polyfluorene with poly[2-methoxy-5-(2'-ethyl-hexyloxy)-1,4phenylenevinylene] (MEH-PPV) as an acceptor in the form of bi-polymer nanoparticles. ${ }^{29}$ However, in this aforementioned work non-functionalized blue and green emitting polymers were utilized to assemble core-shell type nanostructures. The lack of functional groups on the polymers and specific interactions between the layers resulted in less efficient energy transfer and the generation of low quality white light emission.

In this work, we prepared bi-layered and tri-layered white emitting core-shell type nanoparticles by clicking light emitting polymers through alkyne-azide 1,3-dipolar cycloaddition. 
Although white emission can be generated using bi-layered polymer nanoparticles, tri-layered polymer nanoparticles have better white light qualities due to their wider coverage of the spectrum. This approach allowed us to tune the emission colour and the quality of the white light by adjusting the nanoparticle (NP) composition and the energy transfer as well as to obtain stable and shape persistent nanoparticles that remain intact in different solvents because of the cross-linking of functional groups through click chemistry.

\section{Results and discussion}

We have prepared a series of conjugated polymers emitting in the regions of blue, green and red. The molecular structures of these polymers are shown in Fig. 1. As can be seen from their structures, these polymers contain alkyne and azide functional groups that can undergo 1,3-dipolar cycloaddition to form triazole rings while cross-linking the polymers during core-shell type nanoparticle formation.

A blue emitting polymer, PFB-N3, was synthesized from the reaction of a precursor polymer, $\mathrm{PFB}-\mathrm{Br}$, with nucleophilic substitution of sodium azide (ESI, $\dagger$ Fig. S1). The synthesis and characterization of $\mathrm{PFB}-\mathrm{Br}$ were reported in our previous publications. ${ }^{30,31}$ The green emitting polymer, PFBT-P, was synthesized upon functionalization of the precursor polymer, PFBT-Br, with excess propargyl alcohol in the presence of a base and in DMF (ESI, $\dagger$ Fig. S2). PFBTBr was obtained through Suzuki coupling of a fluorene monomer and benzothiadiazole as reported in previous studies. ${ }^{27,32,33}$ Finally, a red emitting polymer, PTH-N3, was synthesized by treating PTHTs with sodium azide in DMF. PTHTs were prepared by the Stille coupling of 2-(2,5-dibromothiophen-3-yl)ethyl-4-methylbenzenesulfonate and 5,5'-bis(tributylstannyl)-2,2'-bithiophene (ESI, $\dagger$ Scheme S1). The synthesis and characterization details of PTH-N3 with NMR, FTIR and GPC are given in the ESI $\dagger$ (Fig. S3-S5).

\section{Bi-polymer nanoparticles}

Here four different designs were investigated in order to obtain white emitting bi-polymer nanoparticles as schematically shown in Fig. 2. The first method involves physically mixing donor and acceptor nanoparticles. For this, first blue and green emitting nanoparticles were prepared using the reprecipitation method. Briefly, blue or green emitting polymers were dissolved in THF and separately injected into rapidly stirring excess water.

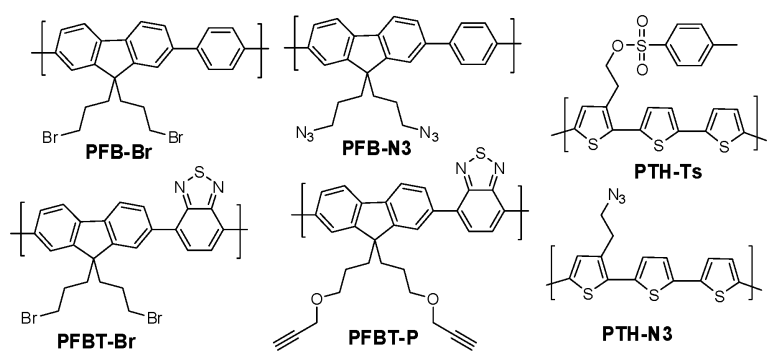

Fig. 1 Molecular structure of polymers used in this work.

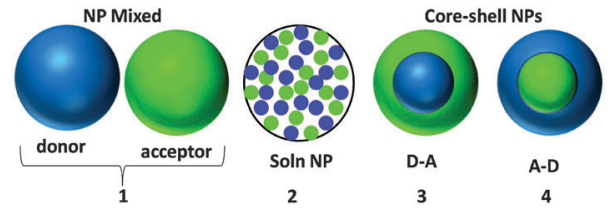

Fig. 2 Illustration of the four bi-polymer NP designs.

After stirring for a while to let the nanoparticles form, THF was removed under reduced pressure. The two nanoparticles were then mixed and denoted as NP mixed. For the second design, PFB-N3 (D) PFBT-P (A) polymers were dissolved in THF and ultrasonicated for $10 \mathrm{~min}$. The solution of polymers was injected into a large excess of rapidly stirring water and the mixture was further sonicated for $40 \mathrm{~min}$ and then THF was removed under reduced pressure to obtain stable bi-polymer nanoparticles. The resulting nanoparticles were denoted as Soln NPs.

In the third method, sequentially formed bi-polymer nanoparticles were designed. Core nanoparticles (PFB-N3) were first prepared (contain azide functional groups and emit blue) and then green emitting polymer (PFBT-P) solution was added (alkyne containing) to form a shell. The resulting bi-polymer nanoparticles, the donor being the core and surrounded by $45 \mathrm{~mol} \%$ acceptor polymers, were denoted as DA45\%. Also DA10\% and DA62\% were prepared similarly with the same donor concentration but varying the acceptor concentrations to be $10 \mathrm{~mol} \%$ and $62 \mathrm{~mol} \%$ of acceptor polymer, respectively. The fourth method is quite the reverse of the third method; core nanoparticles (PFBT-P) were first prepared (alkyne containing and emit green) and then a blue emitting polymer solution (PFB-N3) was added (azide containing) to form a shell. The resulting bi-polymer nanoparticles were denoted as $\mathrm{AD} 45 \%$, the acceptor being the core and surrounded by the donor.

In addition, a catalytic amount of $\mathrm{CuSO}_{4}$ and sodium ascorbate was added to facilitate click reaction in these designs. Another set of these designs were prepared without using $\mathrm{Cu}(\mathrm{I})$ catalysts in order to see whether 1,3-dipolar cycloaddition would still take place.

The size and morphologies of these nanoparticles were determined by SEM, TEM and DLS. Images and DLS histograms are provided in the ESI $\dagger$ (Table S1 and Fig. S6). The average size of the PFBN3, PFBT and PTN3 nanoparticles was determined by DLS measurements to be $64 \mathrm{~nm}, 63 \mathrm{~nm}$ and $80 \mathrm{~nm}$ respectively.

In order to prove that the 1,3-dipolar cycloaddition between azide and alkyne functional groups is taking place in the presence and absence of $\mathrm{Cu}(\mathrm{I})$ catalysts, we recorded the FT-IR spectra of the nanoparticles. The FTIR spectra of some bi-polymer nanoparticles are compared with the spectra of PFBN3 nanoparticles (donor) and PFBT nanoparticles (acceptor) in Fig. 3. The donors, which are functionalized with azide groups, show a strong stretching band of azide at $2098 \mathrm{~cm}^{-1}$. When donor and acceptor nanoparticles were mixed physically (denoted as NP mixed), a broad stretching band of azide was observed as well; this indicates that click reaction does not take place between the functional groups of donor and acceptor nanoparticles when they are mixed physically. Interestingly, in the case of bipolymer nanoparticles 




Fig. 3 FTIR (solid state, $\mathrm{KBr}$ pellet) spectra of bi-polymer nanoparticles. The degree of click reaction was determined to be $97 \%$ for DA $45,95 \%$ for cDA45 and $88 \%$ for DA10.

prepared by click reaction which are catalyzed with $\mathrm{Cu}(\mathrm{I})$ (DA10 and DA45) or without catalysts (cDA45), the azide stretching band at $2098 \mathrm{~cm}^{-1}$ disappeared, indicating the formation of a triazole ring from azides and alkynes. During the core-shell type nanoparticle formation, even in the absence of $\mathrm{Cu}(\mathrm{I})$ catalysts 1,3-dipolar cycloaddition is taking place. The reason could be explained by the appropriate alignment of the alkyne and azide groups because it is expected that most of these groups are located on the surface of the core nanoparticles; this, in turn, allows them to be in close proximity to react faster.

In a bid to synthesize white emitting bi-polymer nanoparticles, four nanostructure designs were investigated. PFBN3 and PFBT polymers were utilized as donor and acceptor pairs, respectively, so as to study energy transfer dependent on nanoparticle morphology and design. ${ }^{32}$ Using FRET, we seek to tune the emission of the hybrid nanoparticles to white, which has potential applications in solid state lighting. First, the photophysical properties of the donor and acceptor polymer nanoparticles were investigated in order to find out whether they are suitable for FRET studies. The absorbance and fluorescence spectra of PFB-N3 (donor) in THF and PFB-N3 nanoparticles in water are given in Fig. 4(a). The donor peak emission wavelength is $\sim 420 \mathrm{~nm}$ when they are dispersed in water to form nanoparticles, while the absorbance and emission bands of PFBT-P nanoparticles (acceptor) are $444 \mathrm{~nm}$ and $544 \mathrm{~nm}$, respectively (Fig. 4b). In Fig. 4d, the overlay of the donor and acceptor spectra shows a strong spectral overlap between the donor emission and absorbance of the acceptor for FRET in the bi-polymer nanoparticles. In addition, PTH-N3 was utilized as a red emitting polymer to design tandem nanoparticles. The peak emission of PTH-N3 is $\sim 620 \mathrm{~nm}$ when dispersed in water to form nanoparticles (Fig. 4c).

In the first bi-polymer nanoparticle design (Fig. 5a), separately formed nanoparticles were mixed in a ratio of $55 \mathrm{~mol} \%$ donor and $45 \mathrm{~mol} \%$ acceptor. With respect to donor and acceptor nanoparticles alone, there was no significant change in the optical properties of the mixed nanoparticles. The emission spectrum shows two peaks at $420 \mathrm{~nm}$ and $544 \mathrm{~nm}$ corresponding to the exact emission of donor and acceptor nanoparticles alone respectively.
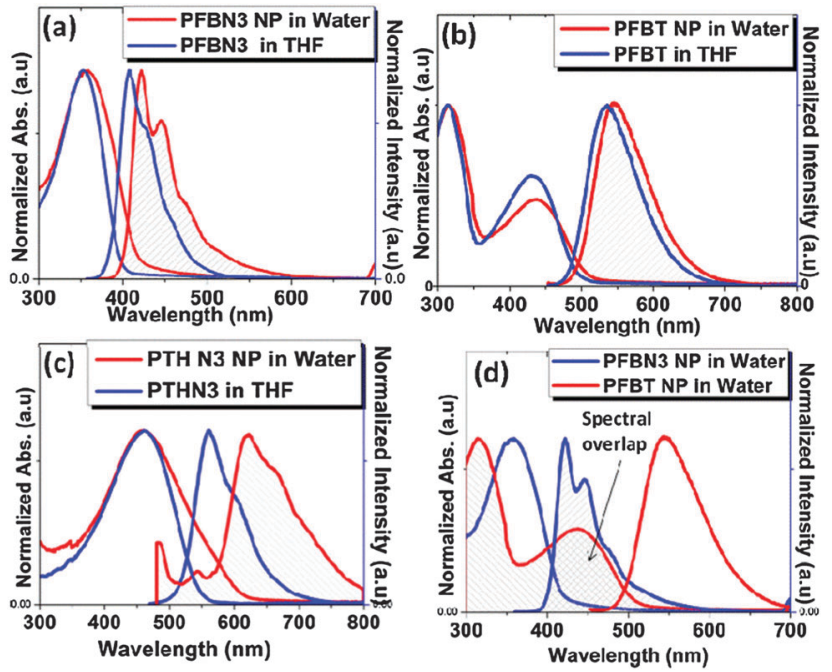

Fig. 4 Absorbance and emission spectra of conjugated polymers in THF and in water: (a) PFBN3 in THF and PFB-N3 CPNs in water, (b) PFBT-P in THF and PFBT CPNs in water, (c) PTH-N3 in THF and PTH-N3 CPNs in water and (d) overlay of spectra showing a strong spectral overlap between PFB-N3 (donor) and PFBT-P (acceptor).

However, the emission at $544 \mathrm{~nm}$ in mixed nanoparticles does suggest that there is energy transfer from the donor to the acceptor. When the emission intensity at $350 \mathrm{~nm}$ excitation is compared with the emission intensity at $435 \mathrm{~nm}$ excitation, a ratio of 0.81 was calculated. This ratio means that there is no strong FRET due to a large distance between the donor and acceptor in solution when the nanoparticles are physically mixed later.

For the second bi-polymer nanoparticle design (Fig. 5b), where solutions of the donor and acceptor were mixed prior to nanoparticle formation, a $5 \mathrm{~nm}$ blue shift was observed along with a 3.2 fold enhancement of the acceptor emission intensity at $350 \mathrm{~nm}$ excitation. Furthermore, the emission due to the donor component was quenched as a result of the strong energy

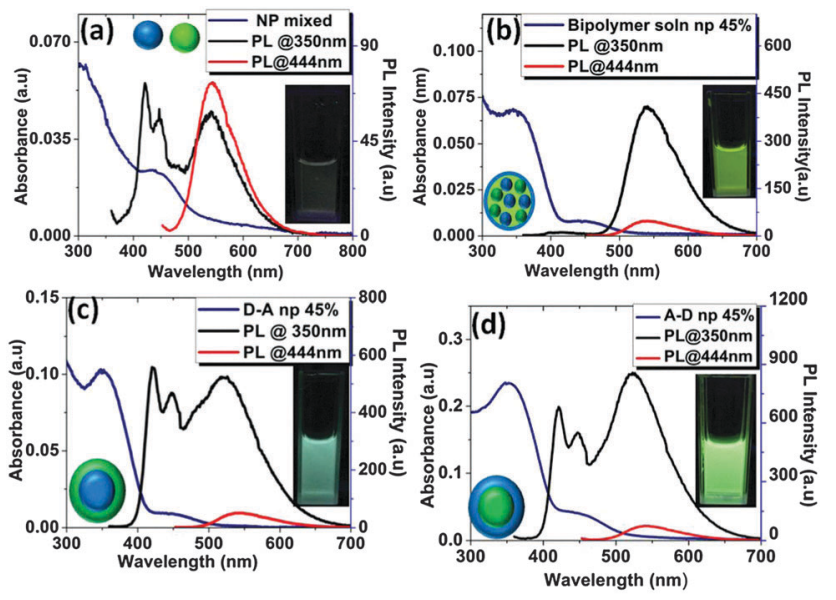

Fig. 5 Absorbance and emission spectra of bipolymer NP dispersion in water: (a) NP mixed (PFB-N3 + PFBT-P NPs), (b) Soln NP (mixing the two polymer solutions prior to NP formation), (c) DA45 NP (PFB-N3/PFBT-P) and (d) AD45 NP (PFBT-P/PFB-N3) ( $\left.\lambda_{\text {ex }}=350 \mathrm{~nm}\right)$. 
transfer from the donor to the acceptor. The almost complete energy transfer using $45 \mathrm{~mol} \%$ acceptor is also obvious from the inset photograph of the dispersion of nanoparticles under a UV lamp (366 nm), showing only a green colour (Fig. 5b).

The third bi-polymer nanoparticle design involves novel core-shell nanoparticles prepared sequentially. Here, the donor forms the core which is coated with $45 \mathrm{~mol} \%$ acceptor (Fig. $5 \mathrm{c}$ ). Excitation of DA45 nanoparticles at donor excitation resulted in $19 \mathrm{~nm}$ blue-shift of the emission of the acceptor. The blue-shift is attributed to an increased $\mathrm{D}-\mathrm{A}$ interaction. The increased oscillating dipole-dipole interactions upon excitation of donors create a nearby electric field, which does not allow the acceptor molecules to find their minima for relaxation so that PFBT-P moieties emit at higher energies. ${ }^{17}$ Furthermore, an efficient energy transfer leading to a 10 fold enhancement of the acceptor emission was observed at donor excitation. The incomplete energy transfer maintains a balance between the emission of both donor and acceptor components, leading to white emission as can be seen in the inset photograph of the DA45 nanoparticles.

Similarly, in the fourth nanoparticle design where the acceptor forms the core surrounded by donor polymers (Fig. 5d), a $21 \mathrm{~nm}$ blue-shift in the acceptor emission was observed as noticed in the opposite design. However, the emission of the acceptor was enhanced by 11 fold with a white-green emission colour. The slightly higher emission intensity of the acceptor is due to efficient energy transfer. Since the acceptor forms the core of the nanoparticles, the loss of energy through reflection and other non-radiative means is negligible.

In the case of nanoparticles prepared without using $\mathrm{Cu}(\mathrm{I})$ catalysts for 1,3-dipolar cycloaddition, there was no significant difference in size as compared to those prepared using catalysts (ESI, $\dagger$ Table S1 and Fig. S7). However, some variations in optical properties were observed. In fact higher FRET efficiencies were invariably observed in uncatalyzed nanoparticles (ESI, $\dagger$ Fig. S9-S11 and Table S2).

The decay curves of catalyzed nanoparticles show a shorter decay lifetime for the donor at $420 \mathrm{~nm}$ as compared to that of only the donor nanoparticles and also a longer decay lifetime for the acceptor emission at $535 \mathrm{~nm}$ as compared to that of the acceptor alone (Fig. 6a and b). These further illustrate the energy transfer between the donor and the acceptor. A similar trend of a decrease in lifetime at $420 \mathrm{~nm}$ and an increase in lifetime at $535 \mathrm{~nm}$ was observed for catalyst-free nanoparticles (Fig. 6c and d). In fact the lifetimes at $535 \mathrm{~nm}$ for catalyst-free nanoparticles are longer than those in the case of catalyzed nanoparticles, which are in agreement with steady-state PL results suggesting an enhanced FRET for the catalyst-free nanoparticles.

The fluorescence lifetimes of the nanoparticles reveal the quantitative measurement of the energy transfer efficiency. The average lifetime of the donor nanoparticle dispersion in water was found to be $0.162 \mathrm{~ns}$ while that of the acceptor nanoparticles was 1.079 ns. Using eqn (1), the energy transfer efficiencies were calculated and the results are tabulated in Table S2 (ESI $\dagger$ ).

$$
\eta=1-\frac{\tau_{\mathrm{DA}}}{\tau_{\mathrm{D}}}
$$
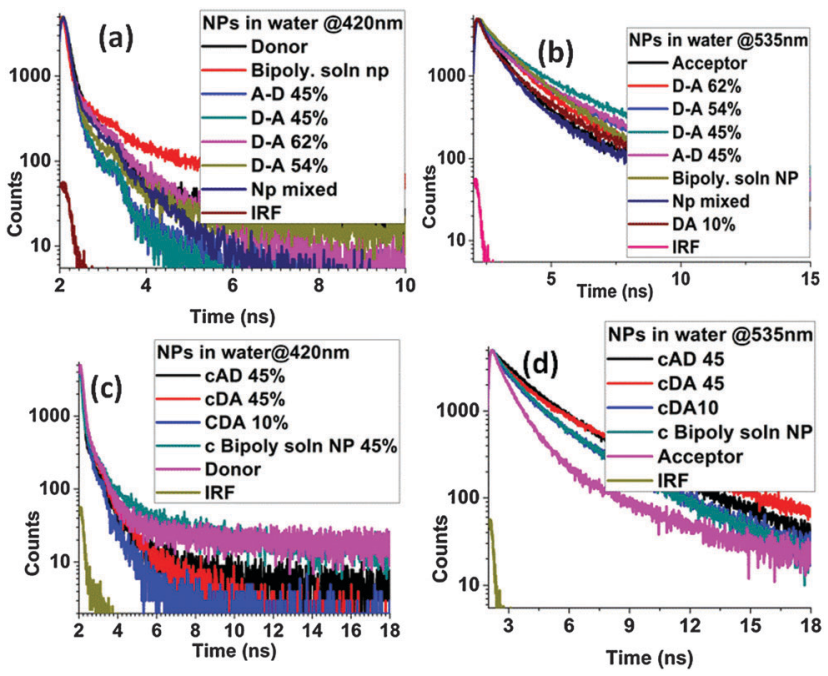

Fig. 6 Biexponentially fitted decay curves of bipolymer nanoparticles: (a) fluorescence lifetime decay curves of catalyzed nanoparticles at $420 \mathrm{~nm}$, (b) at $535 \mathrm{~nm}$, (c) fluorescence lifetime decay curves of catalyst-free nanoparticles at $420 \mathrm{~nm}$ and (d) at $535 \mathrm{~nm}$.

where $\eta$ denotes the energy transfer efficiency, and $\tau_{\mathrm{DA}}$ and $\tau_{\mathrm{D}}$ denote the average lifetime of the donor in the presence and absence of the acceptor, respectively.

However, the energy transfer efficiency of NP mixed was not calculated using eqn (1), because the donor-acceptor pairs are randomly moving in solution with no fixed distance. Nonetheless, all the other nanoparticles bound by a fixed distance could be evaluated using eqn (1). DA62, which contains $62 \mathrm{~mol} \%$ acceptor as the shell, shows the highest energy transfer efficiency of $73.45 \%$. The high energy transfer efficiency of DA62 is due to high acceptor concentration which leads to complete energy transfer from the low content donor core. As for DA45 and cDA45 representing catalyzed bi-polymer nanoparticles and catalyst-free nanoparticles, respectively, the difference in energy transfer efficiency is in agreement with steady-state fluorescence results. The efficiencies of DA45 and cDA45 are $39.5 \%$ and $62.4 \%$ respectively. Again the lower energy transfer efficiency of DA45 compared to cDA45 was due to the quenching effect of copper sulfate used as a catalyst during nanoparticle preparation. Quenching by a catalyst was more pronounced in the nanoparticle design where solutions of the donor and the acceptor were mixed before nanoparticles were prepared. For example a catalyst-free c.Soln nanoparticle has an efficiency of $64.8 \%$ while that of a catalyzed Soln NP is $23.5 \%$. The high quenching in this particular case can be understood from the efficient mixing and distribution of the catalyst throughout the bi-polymer nanoparticles.

For possible application of these bi-polymer nanoparticles in solid state lighting, they should show energy transfer in the solid state as well. A thin film of the bi-polymer nanoparticle was prepared on a quartz surface by drop casting. Interestingly, all the bi-polymer nanoparticles exhibited a high level of energy transfer comparable to that in the nanoparticle dispersion in water (Fig. 7). For NP mixed, where nanoparticles are prepared 


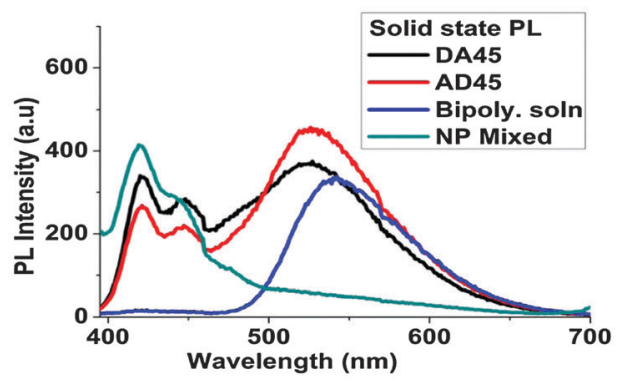

Fig. 7 Solid state photoluminescence of bi-polymer NPs $\left(\lambda_{\mathrm{ex}}=350 \mathrm{~nm}\right)$.

separately from the donor and the acceptor and then mixed physically, very low energy transfer was observed. This result confirms that even at a high concentration of the physical mixture of the donor and the acceptor, they might not be at a favourable distance or orientation for energy transfer to take place. Also, limited exciton diffusion might lead to this observation.

In order to understand the effect of click reaction on the shape and morphology of bi-polymer nanoparticles, sequentially formed nanoparticles were prepared from bromide-functionalized polymers (PFB-Br and PFBT-Br, as the donor and acceptor, respectively) as a control experiment. When compared with azide/alkynefunctionalized polymers which undergo click reaction to form stable nanoparticles, interesting differences in optical properties and morphology were observed. According to DLS and SEM data (ESI, $\dagger$ Fig. S12), when bi-polymer nanoparticles are prepared by click reaction (ADN3), they tend to exhibit smaller, monodispersed and more compact nanoparticles than the nanoparticles prepared from bromide-functionalized polymers (ADBr). Despite the small size differences, ADN3 is $106 \mathrm{~nm}$ while $\mathrm{ADBr}$ is $115 \mathrm{~nm}$, the SEM micrographs showed that ADN3 nanoparticles are more monodispersed.

The optical properties $\mathrm{ADN} 3$ and $\mathrm{ADBr}$ are compared in Fig. 8. Because of the compact nature of ADN3 nanoparticles, which provides a favourable D-A distance for energy transfer, ADN3 demonstrated higher energy transfer than ADBr. Both nanoparticles emit at $420 \mathrm{~nm}$ and $520 \mathrm{~nm}$. The emission at $520 \mathrm{~nm}$ was $24 \mathrm{~nm}$ blue shifted, compared to the acceptor alone.

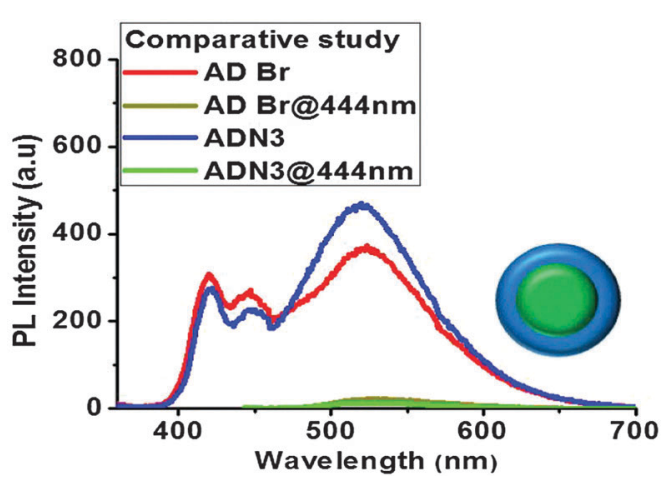

Fig. 8 Photoluminescence spectra of bi-polymer NPs (ADN3) prepared through click reaction and $\mathrm{ADBr}$ prepared from non-functionalized polymers. $\lambda_{\mathrm{ex}}=350 \mathrm{~nm}$ (red and blue lines) and $444 \mathrm{~nm}$ (dark and light green lines).
To demonstrate further that the nanoparticles are stabilized by cross-linking, water dispersions of bi-polymer nanoparticles prepared through click reaction (ADN3) and from non-clickable polymers $(\mathrm{ADBr})$ were evaporated to dryness under reduced pressure. The remaining residues were redispersed in THF and SEM images were taken. While nanoparticles of $\mathrm{ADBr}$ were disintegrated into polymer chains upon treating with THF and the SEM images show no nanoparticles, the SEM micrograph of ADN3 nanoparticles exposed to THF (ESI, $\dagger$ Fig. S13) revealed the presence of nanoparticles, indicating that nanoparticles were not de-folded into separate polymer chains. Their size, however, enlarged from $99 \mathrm{~nm}$ in water to $300 \mathrm{~nm}$ in THF. Moreover, their energy transfer efficiency when they are redispersed in THF falls drastically to an unprecedented low level (ESI, $\dagger$ Table S2). This can be attributed to the swelling of the nanoparticles in THF which is a good solvent, hence, putting the donor and acceptor at an unfavourable distance for efficient FRET to occur. Interestingly, the low energy transfer observed in the solution of bi-polymer nanoparticles in THF which does not occur at all in the case of the solution of $\mathrm{ADBr}$ nanoparticles or bare donor and acceptor polymers in THF suggested that the bi-polymer nanoparticles are covalently bound through click reaction. The triazole rings cross-linking donor and acceptor nanoparticles ensure that even when the nanoparticles are in good solvents (i.e. THF), they only swell to a certain degree but do not disintegrate into their respective polymer chains. Despite the morphology of the bi-polymer nanoparticles in THF are nanoparticle-like, their emission wavelengths shifted to the emission wavelength of the corresponding polymers in THF. The emission of nanoparticles shifted from $420 \mathrm{~nm}$ and $544 \mathrm{~nm}$ to $407 \mathrm{~nm}$ and $535 \mathrm{~nm}$ of the polymer chain respectively. We have also evaporated THF and re-dispersed the nanoparticles in water once again. The re-dispersed nanoparticles were compared with nanoparticles prepared from non-clickable polymers (ADBr NP). All the nanoparticle dispersions in water that were cross-linked through click reaction (both for catalyzed and uncatalyzed) show the restoration of energy transfer. However, the energy transfer in $\mathrm{ADBr}$ nanoparticles was not fully restored (ESI, $\dagger$ Fig. S13).

\section{White-emitting tandem nanoparticles}

Although we have successfully obtained white emission from the bi-polymer core-shell type nanoparticles by tuning the ratio of blue and green emitting polymers, the white colour quality was not at the desirable level. Therefore, in order to generate high quality white light emission, tri-layered or what we call tandem nanoparticles were designed from three polymers (Fig. 9) using four nanostructured design methods as we have discussed for bi-polymer nanostructures. In the first method, 'NP mixed' was designed by preparing nanoparticles from each of the three polymers separately and then mixing them in a certain ratio. In the second method, 'T Sol NP' was prepared by mixing a solution of the three polymers in THF and then nanoparticles were made from the resulting solution. In the third and fourth methods, sequentially formed nanoparticles were prepared; nanoparticles of one polymer are first prepared, 


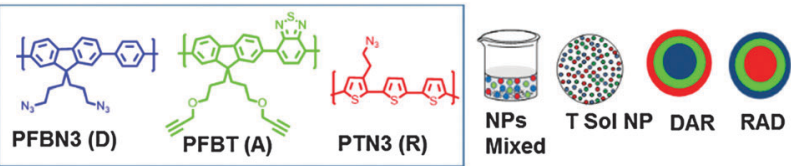

Fig. 9 Schematic representation of tandem nanostructure designs.

followed by coating with the second polymer. The resulting bi-polymer nanoparticles are further coated with the third polymer to obtain sequentially formed tandem nanoparticles.

The size and morphology of the tandem nanoparticles were determined by DLS and SEM (ESI, $\dagger$ Fig. S14-S16). According to the DLS results, the average size of the tandem nanoparticles is $118 \mathrm{~nm}$ which corresponds to a $20 \mathrm{~nm}$ increase in size compared to bi-polymer nanoparticles. The increase in size from $98 \mathrm{~nm}$ in the bi-polymer nanoparticles to $118 \mathrm{~nm}$ in the tandem nanoparticles is a clear indication of the successful formation of tri-layered nanoparticles. The SEM micrographs of the tandem nanoparticles show spherical particles with no formation of aggregates. Fig. 10 shows SEM and TEM images of DAR $4 \%$ NPs.

The energy transfer study of the four tandem nanoparticle designs are presented in Fig. S17 (ESI $\dagger$ ). Each of the tandem nanoparticles contains an equal ratio of blue, green and red polymers for comparison. They all contain $58 \mathrm{~W} \%$ blue, $24 \mathrm{~W} \%$ green and $18 \mathrm{~W} \%$ red polymers.

In the first method, 'NP mixed' shows an emission spectrum similar to that of the individual nanoparticles. Some energy transfer from blue to green and then to the red polymer could be observed. However, the energy transfer was inefficient owing to the large distance between the nanoparticles in solution. The dominance of the donor component at $420 \mathrm{~nm}$ results in a blue colour. In the second design, where solutions of three polymers were simultaneously used to prepare 'T Sol NPs', a complete energy transfer from the donor to the acceptor and finally to the red polymer was observed. This result is similar to the results observed for the bi-polymer nanoparticles mentioned earlier. All the blue and green polymer components of the spectrum at $420 \mathrm{~nm}$ and $535 \mathrm{~nm}$, respectively, have been transferred to the red polymer component at $595 \mathrm{~nm}$. The emission of the red polymer was enhanced 3 fold through energy transfer compared to the emission of the red polymer at its excitation wavelength.

In the third design denoted as DAR NPs, blue polymer nanoparticles were coated with the green polymer followed by

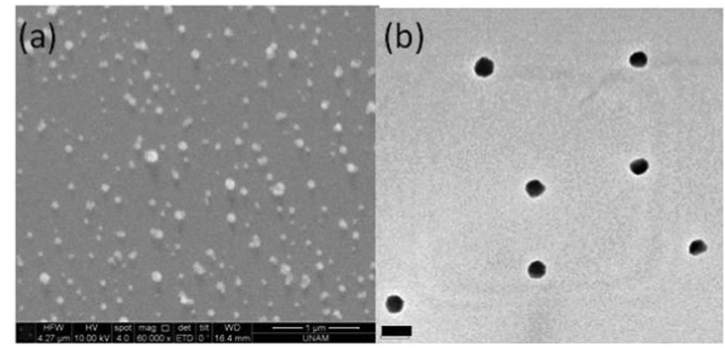

Fig. 10 Size and morphology of DAR 4\% NPs 101 nm: (a) SEM micrograph (scale $1 \mu \mathrm{m}$ ) and (b) TEM micrograph (scale $0.2 \mu \mathrm{m}$ ). the red polymer. DAR NPs show high energy transfer to the green polymer component with a $10 \mathrm{~nm}$ blue shift. The emission intensity of the blue polymer component at $420 \mathrm{~nm}$ was very low due to the efficient energy transfer to acceptors. The emission intensity of the green polymer was 8 times higher at $350 \mathrm{~nm}$ excitation than its own excitation at $444 \mathrm{~nm}$.

Finally, for the fourth design denoted as RAD NPs, red polymer nanoparticles were coated with the green polymer followed by the blue polymer. The formation of sequentially formed tandem nanoparticles was evidenced from the spectral changes. The distinct emission of the three polymers can be seen at the $350 \mathrm{~nm}$ excitation wavelength. However, the emission intensities of the blue and green polymers are very low compared to the red polymer due to energy transfer. Furthermore, the emission of the red polymer was $35 \mathrm{~nm}$ blue-shifted compared to polythiophene nanoparticles alone. In addition, the emission intensity of the red polymer component of the RAD NPs is 6.6 times higher than the emission intensity of the red polymer nanoparticles at its own excitation wavelength. Such amplified emission is attributed to high energy transfer and prevention of aggregated quenching of the red polymer when doped with other polymers.

Among the four nanostructured designs studied, DAR shows an emission colour that resembles white light. As a result DAR NP design was adapted to prepare a set of tandem white emitting nanoparticles. To further tune the emission of DAR, the $42 \mathrm{~W} \%$ green and red emitting polymer components were limited to $12 \mathrm{~W} \%$ to obtain new white emitting tandem nanoparticles denoted as DAR $12 \%$. DAR $12 \%$ was prepared with $88 \mathrm{~W} \%$ blue, $9 \mathrm{~W} \%$ green and $3 \mathrm{~W} \%$ red polymers. The green and red polymers in DAR $12 \%$ add up to $12 \%$ by weight. In Fig. 11a, the emission spectrum of DAR $12 \%$ showed the distinct emissions of the three polymers. Due to incomplete energy transfer and the wide coverage of the visible region, white emission was achieved.

Moreover, the emission of DAR $12 \%$ NPs was also tuned by further limiting the green and red polymer components to give DAR 6\% and DAR 4\% NPs. The reduction of the blue and green polymer components from $12 \%$ to $4 \%$ by weight is expected to induce incomplete energy transfer and hence the generation of white emission. In DAR 6\%, the compositions of polymers are $94 \mathrm{~W} \%$ blue, $4.5 \mathrm{~W} \%$ green and $1.5 \mathrm{~W} \%$ red polymers. For DAR $4 \%$ where the compositions are $96 \mathrm{~W} \%$ blue, $3 \mathrm{~W} \%$ green and $1 \mathrm{~W} \%$ red polymers, the white emission was comparable to that of DAR 6\% NPs (Fig. 11b). The quality of the white emission from DAR $4 \%$ was the best achieved (Fig. 11c). The average size of DAR $4 \%$ NPs was determined to be $101 \mathrm{~nm}$ according to DLS measurements.

The fluorescence decay curves of the tandem nanoparticles at $420 \mathrm{~nm}$ show that NP mixed has a longer lifetime (0.158 ns) compared to the other tandem nanoparticle designs, which range from $0.048-0.126 \mathrm{~ns}$. This indicates an inefficient energy transfer in nanoparticle mixed. Furthermore, the fluorescence decay curves at $535 \mathrm{~nm}$ (Fig. 12b) illustrate energy transfer in the tandem nanoparticles except for NP mixed which has the shortest lifetime at $535 \mathrm{~nm}$ (ESI, $\dagger$ Table S3). 

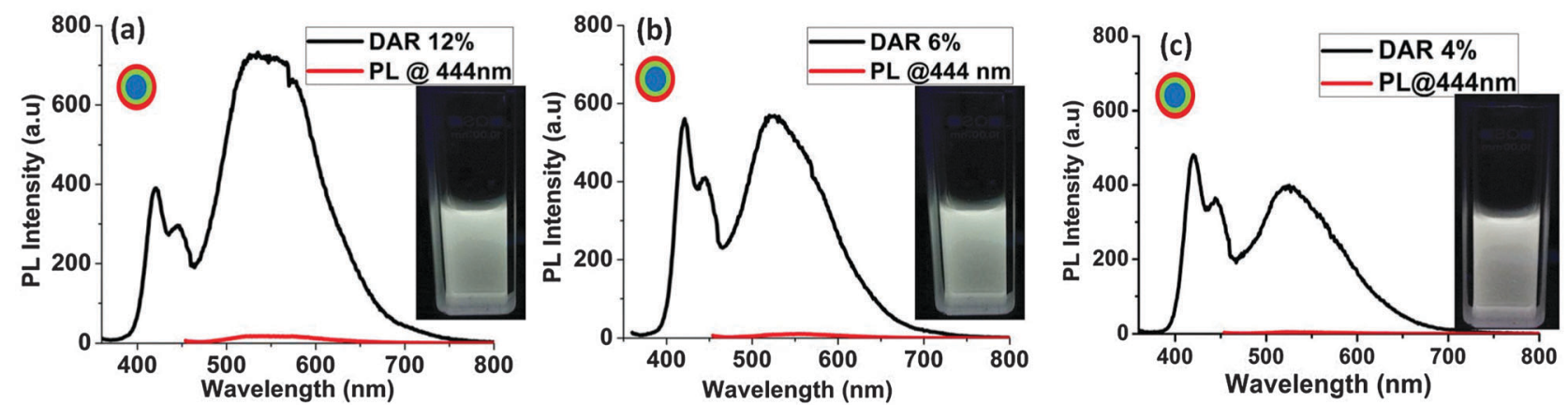

Fig. 11 Emission spectra of (a) DAR 12\% NPs $\left(\lambda_{\mathrm{ex}}=350 \mathrm{~nm}\right.$ ), (b) DAR $6 \%$ NPs and (c) DAR $4 \%\left(\lambda_{\mathrm{ex}}=350 \mathrm{~nm}\right)$. The red lines represent the excitation at $444 \mathrm{~nm}$. Insets show the photographs of nanoparticle dispersions in water taken under $365 \mathrm{~nm}$ UV light.
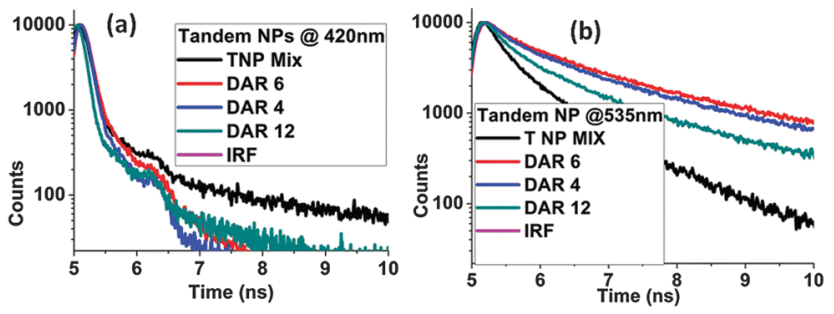

Fig. 12 Biexponentially fitted decay curves of tandem nanoparticles: (a) fluorescence lifetime decay curves of tandem NPs at $420 \mathrm{~nm}$ and (b) at $535 \mathrm{~nm}$.

To realize proof-of-concept white light-emitting diodes (LEDs) employing tandem nanoparticles, the first step was the evaluation of the colour quality of tandem nanoparticle dispersions. Our calculations revealed that DAR $4 \%$ dispersion exhibits the best colour rendering index (CRI), reaching 58.3 with the colour quality scale (CQS) reaching 74.1, which indicate the capability of the light source to render the real colours of the objects, along with a reasonable correlated colour temperature (CCT) of $8716 \mathrm{~K}$.

Subsequent to the selection of the tandem nanoparticles for white LED applications, tandem nanoparticle films were prepared by dissolving $120 \mathrm{mg}$ of poly(vinyl)pyrolidone in $2 \mathrm{~mL}$ of nanoparticle dispersion. Later, $0.7 \mathrm{~mL}$ of this mixture was drop-casted on glass and dried at $c a .60{ }^{\circ} \mathrm{C}$. Finally, the prepared film was placed on top of a LED emitting at $380 \mathrm{~nm}$ and the emission spectrum was recorded using a Maya 2000 spectrometer at varying current levels. Corresponding emission spectra are presented in Fig. 13 together with the corresponding CRI, CQS, CCTs and corresponding Commission Internationale de L'Eclairage (CIE) coordinates (full coordinates are given in the ESI, $\dagger$ Table S4).

This LED exhibited CCTs between 4910 and $4952 \mathrm{~K}$, which fall in the region of frequently used white light sources. Additionally, the CRI values varied between 71.9 and 72.4, and CQS values varied between 78.4 and 79.0 at varying applied currents. We observe that higher photon flux from the LED chip at higher currents does not cause any significant change in the colour quality of the final device.

The colour rendition performance of this nanoparticle integrated LED becomes comparable with YAG phosphor integrated LEDs.

DAR $4 \%$ nanoparticles using $\mathrm{Cu}(\mathrm{I})$-catalyst integrated LEDs were also prepared following the same procedure as the DAR $4 \%$ (without using a catalyst) tandem white nanoparticles. These LEDs exhibited inferior colour rendering performance compared to DAR $4 \%$ white nanoparticle LEDs (ESI, $†$ Fig. S18). This is mainly because of the lower intensity of the blue part in the spectrum compared to the previous case. This variation in the spectrum mainly occurs due to the presence of the $\mathrm{Cu}$ ions causing quenching. This lower intensity also affects the perceived colour to a greenish shade while DAR $4 \%$ is much closer to the white point on the chromaticity diagram (ESI, $\dagger$ Fig. S19).

\section{Experimental}

All experimental details regarding the synthesis and characterization of polymers and nanoparticles are provided in the ESI. $\dagger$
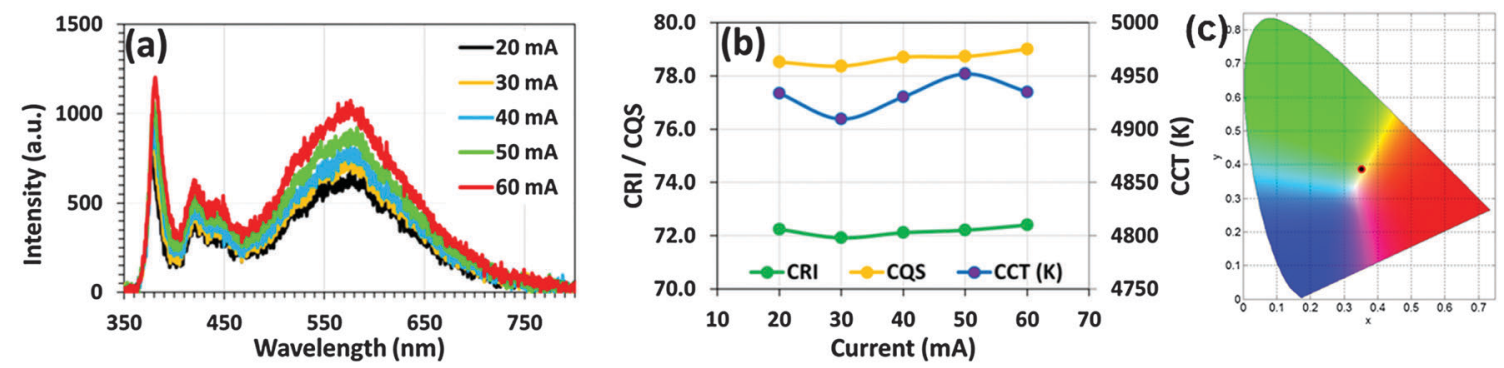

Fig. 13 (a) The spectra and (b) CRI, CQS, and CCT of the tandem nanoparticle integrated white LED at varying current levels and (c) corresponding CIE coordinates. 


\section{Conclusions}

Four nanoparticle designs were utilized in this work. The first approach involved preparing PFBN3 and PFBT nanoparticles separately and mixing them in a determined ratio. In the second approach, solutions of PFBN3 and PFBT are mixed before nanoparticle formation; the nanoparticles are prepared from a mixture of two polymer solutions. In the third and fourth approaches, sequentially formed nanoparticles were designed. In these designs, the third one, in which the core is made out of the donor and the shell is composed of acceptor polymers, was found to be the most efficient one allowing us to tune the emission colour and the quality of the white light by adjusting the nanoparticle composition and the energy transfer. Using this approach, we were able to prepare bi-layered and trilayered core-shell type nanoparticles by clicking polymers with appropriate functional groups through alkyne-azide 1,3-dipolar cycloaddition to obtain stable and shape persistent nanoparticles that remained intact in different solvents because of the crosslinking of functional groups through click chemistry. Interestingly, even in the absence of $\mathrm{Cu}(\mathrm{I})$-catalysts alkyne-azide 1,3-dipolar cycloaddition took place because of the appropriate alignment of the alkyne-azide functional groups in these nanoparticles.

Although bi-layered polymer nanoparticles were capable of producing white emission, tri-layered polymer nanoparticles or tandem nanoparticles turned out to display better white light qualities due to their wider coverage of the spectrum. Moreover, LEDs fabricated using tandem nanoparticles in the absence of $\mathrm{Cu}(\mathrm{I})$-catalysts exhibited superior colour rendering performance compared to the one prepared from $\mathrm{Cu}(\mathrm{I})$-catalyzed nanoparticles.

These tandem nanoparticles can be used as colour converters to realize a white LED acquiring a colour quality comparable to existing phosphor based white light emitting diodes.

\section{Acknowledgements}

We acknowledge TUBITAK-TBAG 112 T704 and COST Action TD1004 (Theragnostics Imaging and Therapy: an Action to Develop Novel Nanosized Systems for Imaging-Guided Drug Delivery).

\section{Notes and references}

1 K. T. Kamtekar, A. P. Monkman and M. R. Bryce, $A d v$. Mater., 2010, 22, 572-582.

2 G. M. Farinola and R. Ragni, Chem. Soc. Rev., 2011, 40, 3467-3482.

3 M. C. Gather, A. Köhnen and K. Meerholz, Adv. Mater., 2011, 23, 233-248.

4 B. W. D'Andrade and S. R. Forrest, Adv. Mater., 2004, 16, 1585. 5 J. Kido, M. Kimura and K. Nagai, Science, 1995, 267, 1332-1334.
6 N. T. Kalyani and S. J. Dhoble, Renewable Sustainable Energy Rev., 2012, 16, 2696-2723.

7 H. J. Song, G. J. Shin, K. H. Choi, S. Lee and D. K. Moon, Synth. Met., 2014, 190, 1-7.

8 S. Reineke, F. Lindner, G. Schwartz, N. Seidler, K. Walzer, B. Lussem and K. Leo, Nature, 2009, 459, 234-238.

9 M. J. Park, J. Lee, I. H. Jung, J. H. Park, D. H. Hwang and H. K. Shim, Macromolecules, 2008, 41, 9643-9649.

10 Z. Zhang, P. Yan, S. Yue, Y. Chen, Q. Wu, D. Qu, R. Guo, P. Wang, Y. Zhao and S. Liu, Org. Electron., 2013, 14, 2172-2176.

11 Y. Sun, N. C. Giebink, H. Kanno, B. Ma, M. E. Thompson and S. R. Forrest, Nature, 2006, 440, 908-912.

12 G. Schwartz, M. Pfeiffer, S. Reineke, K. Walzer and K. Leo, Adv. Mater., 2007, 19, 3672-3676.

13 H. Sasabe and J. Kido, J. Mater. Chem. C, 2013, 1, 1699-1707. 14 T. Ahn and H. K. Shim, Macromol. Chem. Phys., 2001, 202, 3180. 15 I. O. Huyal, T. Ozel, U. Koldemir, S. Nizamoglu, D. Tuncel and H. V. Demir, Opt. Express, 2008, 16, 1115-1124.

16 I. O. Huyal, U. Koldemir, T. Ozel, H. V. Demir and D. Tuncel, J. Mater. Chem., 2008, 18, 3568-3574.

17 L. Ying, C. L. Ho, H. Wu, Y. Cao and W. Y. Wong, Adv. Mater., 2014, 26, 2459-2473.

18 J. R. Lakowicz, Principles of Fluorescence Spectroscopy, Springer, New York, 2006.

19 J. Liu, L. Li and Q. B. Pei, Macromolecules, 2011, 44, 2451.

20 H. Wu, L. Ying, W. Yang and Y. Cao, Chem. Soc. Rev., 2009, 38, 3391-3400.

21 Q. Wang and D. Ma, Chem. Soc. Rev., 2010, 39, 2387-2398.

22 J. Liu, Q. Zhou, Y. Cheng, Y. Geng, L. Wang, D. Ma, X. Jing and F. Wang, Adv. Mater., 2005, 17, 2974-2978.

23 C. Y. Chuang, P. I. Shih, C. H. Chien, F. I. Wu and C. F Shu, Macromolecules, 2007, 40, 247-252.

24 C. F. Huebner, R. D. Roeder and S. H. Foulger, Adv. Funct. Mater., 2009, 19, 3604-3609.

25 T. Kietzke, D. Neher, K. Landfester, R. Montenegro, R. Güntner and U. Scherf, Nat. Mater., 2003, 2, 408-412.

26 I. O. Huyal, T. Ozel, D. Tuncel and H. V. Demir, Opt. Express, 2008, 16, 13391-13397.

27 E. J. Park, T. Erdem, V. Ibrahimova, S. Nizamoglu, H. V. Demir and D. Tuncel, ACS Nano, 2011, 5, 2483-2493.

28 Y. Hou, S. Cao, X. Li, B. Wang, Y. Pei, L. Wang and Z. Pei, ACS Appl. Mater. Interfaces, 2014, 6, 16909-16917.

29 I. O. Ozel, T. Ozel, H. V. Demir and D. Tuncel, Opt. Express, 2010, 18, 670-684.

30 B. Baykal, V. Ibrahimova, G. Er, E. Bengü and D. Tuncel, Chem. Commun., 2010, 46, 6762-6764.

31 V. Ibrahimova, M. E. Kocak, A. M. Onal and D. Tuncel, J. Polym. Sci., Part A: Polym. Chem., 2013, 51, 815-823.

32 T. Erdem, V. Ibrahimova, D. W. Jeon, I. H. Lee, D. Tuncel and H. V. Demir, J. Phys. Chem. C, 2013, 117, 18613-18619.

33 V. brahimova, S. Ekiz, O. Gezici and D. Tuncel, Polym. Chem., 2011, 2, 2818-2824. 